\begin{tabular}{|c|c|}
\hline Title & New sample cell configuration for wide frequency dielectric spectroscopy : DC to radio frequencies \\
\hline Author(s) & Nakanishi, Masahiro; Sasaki, Y asutaka; Nozaki, Ryusuke \\
\hline Citation & $\begin{array}{l}\text { Review of Scientific Instruments, 81(12), } 123902 \\
\text { https://doi.org/10.1063/1.3518940 }\end{array}$ \\
\hline Issue Date & 2010-12 \\
\hline Doc URL & http:/hdl.handle.net/2115/44911 \\
\hline Rights & $\begin{array}{l}\text { Copyright } 2010 \text { A merican Institute of Phy sics. This article may be downloaded for personal use only. Any other use } \\
\text { requires prior permission of the author and the A merican Institute of Physics. The following article appeared in Rev. } \\
\text { Sci. Instrum. } 81,123902 \text { (2010) and may be found at https:/dx.doi.org/10.1063/.3518940 }\end{array}$ \\
\hline Type & article \\
\hline File Information & RSI81-12_123902.pdf \\
\hline
\end{tabular}

Instructions for use 


\title{
New sample cell configuration for wide-frequency dielectric spectroscopy: DC to radio frequencies
}

\author{
Masahiro Nakanishi (中西真大), ${ }^{a)}$ Yasutaka Sasaki (佐々木康隆), and Ryusuke Nozaki \\ (野岦龍介) \\ Department of Physics, Faculty of Science, Hokkaido University, Sapporo, Hokkaido 060-0810, Japan
}

(Received 2 September 2010; accepted 30 October 2010; published online 27 December 2010)

\begin{abstract}
A new configuration for the sample cell to be used in broadband dielectric spectroscopy is presented. A coaxial structure with a parallel plate capacitor (outward parallel plate cell: OPPC) has made it possible to extend the frequency range significantly in comparison with the frequency range of the conventional configuration. In the proposed configuration, stray inductance is significantly decreased; consequently, the upper bound of the frequency range is improved by two orders of magnitude from the upper limit of conventional parallel plate capacitor $(1 \mathrm{MHz})$. Furthermore, the value of capacitance is kept high by using a parallel plate configuration. Therefore, the precision of the capacitance measurement in the lower frequency range remains sufficiently high. Finally, OPPC can cover a wide frequency range $(100 \mathrm{~Hz}-1 \mathrm{GHz})$ with an appropriate admittance measuring apparatus such as an impedance or network analyzer. The OPPC and the conventional dielectric cell are compared by examining the frequency dependence of the complex permittivity for several polar liquids and polymeric films. () 2010 American Institute of Physics. [doi:10.1063/1.3518940]
\end{abstract}

\section{INTRODUCTION}

Measurements of the complex permittivity of materials over a wide frequency range have attracted interest in areas of both basic and applied science, ${ }^{1}$ including glass transitions, polymers, aqueous solutions, liquid crystals, and ferroelectric materials. For all fields, it is important to be able to evaluate the dielectric characteristics of a material simply and precisely over a wide frequency range. Recently, electronic technology has advanced remarkably, and sophisticated instruments can now be used for measurements. The latest impedance analyzers cover a wide frequency range from $10 \mathrm{~Hz}$ to $100 \mathrm{MHz}$ (e.g., Wayne Kerr Electronics 65120B, $20 \mathrm{~Hz}-120 \mathrm{MHz} ;{ }^{2}$ Agilent technology 4294A, $40 \mathrm{~Hz}-$ $110 \mathrm{MHz} ;{ }^{3}$ Solartron $\left.1260,10 \mu \mathrm{Hz}-32 \mathrm{MHz}\right){ }^{4}$ In a practical sense, however, full frequency range measurements remain difficult to perform, primarily because of the lack of an optimized sample cell. Generally, in the low frequency (LF) region (below $1 \mathrm{MHz}$ ), the sample under test is sandwiched between two plane electrodes to which lead wires are connected. Using this method, systematic error increases with increasing frequency due to the inductance component. This limits the applicability of the sample cell. On the other hand, for measurements in the high frequency (HF) region (above $1 \mathrm{MHz}$ ), coaxial line shaped sample cells are often used. Because the inductance component of such cell is very small, the applicable frequencies are extended to the HF region. However, the capacitance of such cells is also typically small, and thus the applicability of this method is limited only to the HF region. For these reasons, wide frequency measurements covering both LF and HF regions in a single sweep are still difficult.

In this paper, we present a newly developed sample cell called the outward parallel plate cell (OPPC), which is intended for wide frequency dielectric measurement of liquids

a)Electronic mail : nakanishi@dielectrics.sci.hokudai.ac.jp. and film materials. The capacitance of the proposed sample cell is very large whereas the inductance is very small. The details of this method and its advantages over conventional technique are discussed.

\section{DRAWBACKS TO CONVENTIONAL METHOD AND REQUIREMENTS FOR NEW METHOD}

In addition to the device under test (DUT), parasitic components always exist within the circuit. The most appealing ones are the shunt capacitor and series inductor associated with the DUT. The former originates from the unexpected electric field between high electrode and low electrode. The latter is due to the induced magnetic field around the wire. The impedance of the capacitor, $Z_{C}$, is given as $1 / j \omega C$, and the impedance of the inductor, $Z_{L}$, is $j \omega L$, where $j$ is $\sqrt{-1}$, $\omega$ is angular frequency, $C$ is capacitance, and $L$ is inductance. Therefore, the relation between the impedance of the DUT and that of the parasitic components is frequency dependent.

When a capacitor is set as the DUT, the relation between the impedance of the DUT and that of the parasitic capacitor is identical at all frequencies. In the LF region, their impedances are much greater than that of the parasitic inductor; accordingly, the effects of parasitic inductor are negligible and only the contribution from capacitors needs to be considered. Because the impedance of a capacitor is proportional to the reciprocal frequency, the impedance becomes considerably larger in the LF region. Such high impedance suppresses the current through the DUT and therefore decreases the accuracy of measurement. For measurements in the LF region, the large capacitance basically gives better accuracy. For example, in the measurement of a capacitor using the Solartron 1260 and Agilent 4294A, capacitance of greater than $100 \mathrm{pF}$ (Ref. 4) and $1 \mathrm{nF}$ (Ref. 3) is required for 1\% accuracy at $100 \mathrm{~Hz}$, respectively. For this reason, increasing the capacitance of the sample cell is essential in the LF region. 
On the other hand, because the impedance of the inductor is proportional to frequency, its effect becomes apparent only in the $\mathrm{HF}$ region. Above $1 \mathrm{MHz}$, parasitic inductance due to the lead wire causes a systematic error. For example, a set of four 1-m coaxial cables for the standard four-terminal pair method gives inductance of $1000 \mathrm{nH}$ and thus impedance of $6 \Omega$ at $1 \mathrm{MHz}$. At this frequency, the capacitance of typical sample cell, $100 \mathrm{pF}$, gives an impedance value of $1500 \Omega$. From this estimation, the significance of the parasitic inductance has been clarified. The inductance is generally proportional to the length of the components. Additionally, the shape of the component is also essential. For instance, even a few centimeters of an exposed wire pair connected with the sample cell leads to an inductance of $100 \mathrm{nH}$. For measurements at over $1 \mathrm{MHz}$, a non-coaxial structure gives rise to high parasitic inductance. Therefore, keeping the coaxial structure until the end of the whole circuit is essential for HF measurements.

Basically, two types of sample cells, namely, coaxial and parallel plate sample cells, are conventionally employed. ${ }^{1,5,6}$ Generally, the former is used in the HF region, such as radio frequencies, ${ }^{7-10}$ and the latter is used in the LF region. ${ }^{10-12}$ For coaxial cells, it is straightforward to keep a coaxial structure up to the end of the whole circuit and the parasitic inductance can be minimized at an extremely low level; typically, less than $1 \mathrm{nH}$. The coaxial structure is therefore highly suitable for HF measurements. However, because of its cylindrical shape, it is difficult to make a large capacitance cell, typically less than $5 \mathrm{pF}$, and therefore the coaxial structure cannot be used in the LF region. On the other hand, the parallel plate type cell is suitable for increasing capacitance because it is simple to decrease the gap between electrodes and to increase an area of the electrode, where capacitance greater than $10 \mathrm{pF}$ can be easily attained. This gives an advantage for LF measurements. It should be noted that this configuration is convenient not only for liquid but also for film samples.

For measurements covering a wide frequency region, it is important to consider the advantages of both coaxial and parallel plate type cells. This is, however, very difficult; in fact, such cells with the advantages of both types have not yet been developed. To overcome the difficulty of implementing such a cell, the principles of the following two excellent HF measurement techniques can be partly used. One is the sample cell proposed by Novocontrol, ${ }^{13}$ and the other is the sample cell proposed by Nozaki and coworkers. ${ }^{14}$ The frequency ranges covered by each cell are slightly different, but they both take advantage of using the structure of the APC7 connector. APC7 has a large diameter in comparison with other high-frequency connectors. In these cells, a parallel plate-like structure is included within the cavity of APC7. By using this structure, good propagation characteristics with small inductance can be attained. It is, however, impossible to increase the capacitance because of the spatial limitation of the cavity in the APC7.

\section{CELL DEVELOPMENT}

The cell presented in this paper has a parallel plate shape in addition to topologically maintaining a coaxial structure extending the electrodes toward the outward direction. This

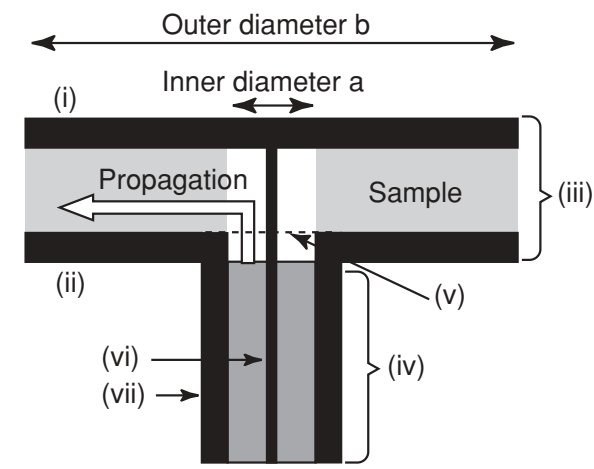

FIG. 1. Conceptual framework of OPPC (black parts correspond to the conductor): (i) upper electrode, (ii) lower electrode, (iii) parallel plate capacitor, (iv) connector and coaxial cable, (v) reference plane of calibration, (vi) inner conductor, and (vii) outer conductor. Arrow shows the propagation direction of incident electromagnetic wave. In this figure, the gap separation between two electrodes (i) and (ii) is overly depicted: in the actual OPPC, the gap separation is of the order of $100 \mu \mathrm{m}$ whereas diameter of the electrodes is of the order of $10 \mathrm{~mm}$. Sample liquid is kept by surface tension. White part at the center of this figure is not filled with sample.

shape increases the capacitance to a high level while the inductance remains low. The newly developed cell is called the OPPC.

Figure 1 shows the conceptual framework of the OPPC. The cell consists of two parallel plate electrodes, namely, the upper electrode (i) and lower electrode (ii). The electrodes are shaped as discs to maintain good symmetry. The bottom center part of the lower electrode is connected to the coaxial cable with a certain connector (iv). The upper electrode is connected to the high-voltage inner conductor (vi) and the lower electrode is connected to the low-voltage (signal ground) outer conductor (vii). An incident electromagnetic wave propagates from the center toward the outside (represented by the arrow). From this propagation characteristic, the structure of the OPPC can be interpreted to be topologically equivalent to that of a coaxial cable, where the configuration of the OPPC could be continuously changed into coaxial cable. This is one reason for the good performance of the OPPC at high frequencies. It should be noted that such a concept is not applicable to a conventional parallel plate capacitor.

The capacitor lies between discs of diameters $a$ and $b$. Although diameter $a$ depends on the type of connector used, the OPPC with APC7 connector exhibits the best characteristics (as explained in the next paragraph). In the case of using APC7, the diameter $a$ is $7 \mathrm{~mm}$, which is the outer diameter of the APC7 connector. Although there is no specific limitation for the diameter $b$, an overly large diameter would cause resonance due to a capacitor and inductor being in series in a circuit, where the resonance frequency is given by

$$
f=1 / 2 \pi \sqrt{L C} .
$$

Because this would degrade the precision of the measurement, the diameter should be chosen by carefully considering these requirements. The diameter $b$ of our cell was $50 \mathrm{~mm}$ and the gap separating the two electrodes, $d$, was about $100 \mu \mathrm{m}$. The capacitance and inductance of the OPPC can be calculated from these parameters by neglecting the contribution from the fringe effect. The capacitance $d C$ and the 


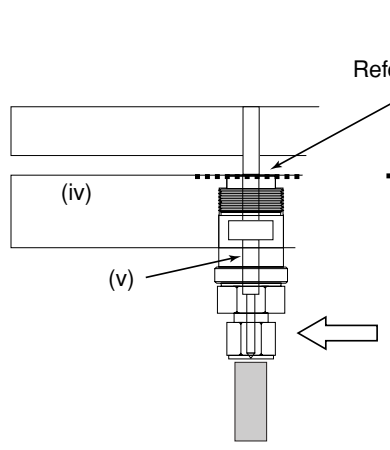

(c)

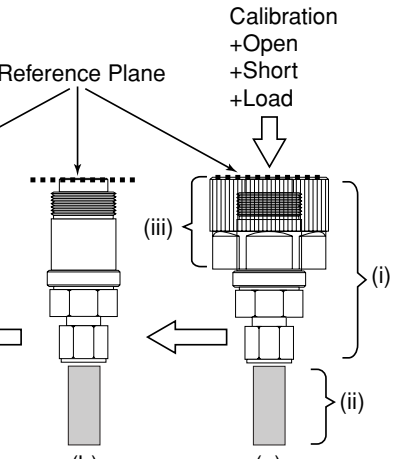

(b)

(a)
FIG. 2. Calibration setup for OPPC: (i) APC7 connector, (ii) coaxial cable, (iii) metallic part covering the outer conductor of APC7, (iv) electrodes of OPPC, and (v) inner conductor of APC7.

inductance $d L$ of the faced rings within radius from $r$ to $r+d r$ and with gap separation $d$ are given by $d C=$ $\left(2 \pi \varepsilon_{0} r / d\right) d r$ and $d L=\left(\mu_{0} d / 2 \pi r\right) d r$, respectively. Integrating them from inner diameter to outer diameter, the capacitance $C$ and the inductance $L$ of the OPPC are calculated as follows:

$$
\begin{aligned}
& C=\int_{a / 2}^{b / 2} \varepsilon_{0} \frac{2 \pi}{d} r d r=\varepsilon_{0} \frac{\pi\left(b^{2}-a^{2}\right)}{4 d}, \\
& L=\int_{a / 2}^{b / 2} \frac{\mu_{0} d}{2 \pi r} d r=\mu_{0} \frac{d}{2 \pi} \ln (b / a),
\end{aligned}
$$

where $\varepsilon_{0}$ and $\mu_{0}$ are the dielectric constant and magnetic permeability of vacuum, respectively. Substituting these parameters into the above equations yields $C=170 \mathrm{pF}$ and $L=39 \mathrm{pH}$. The inductance is suppressed at an extremely low level, whereas the capacitance is kept sufficiently high. This is due to the topologically equivalent structure to a coaxial line. According to Eq. (1) the resonance frequency is estimated to be $1.9 \mathrm{GHz}$ and this limits the applicability of the OPPC by around this frequency.

In the present study, the APC7 connector is used at the contact between the OPPC and coaxial cable. This results in good electrical properties because of the flat contact plane of the APC7, and improves the calibration performance. Figure 2 shows the calibration procedure of the OPPC. The APC7 (i) connected to the end of the coaxial cable (ii) is shown on the right (a). First, commercially available calibration kits (open, short, and load) are attached on the top of the APC7. In this procedure, the reference plane is set on the top end of the APC7 (dotted line). After the calibration, the metallic part covering the outer conductor (iii) is removed (b), and subsequently the electrodes of OPPC (iv) are attached (c), instead of the metallic part. As explained above, the reference plane is on the top end of the APC7 (dotted line), and the APC7 has a flat contact structure. Therefore, only the contribution from the OPPC electrodes can be measured without



FIG. 3. Detail of the OPPC design: (i) upper supporting plate, (ii) upper electrode, (iii) movable center pin, (iv) micrometer head, (v) lower supporting plate, (vi) lower electrode, (vii) APC7 connector, and (viii) spacer. The movable center pin is to be contacted with the inner conductor of APC7 connector by adjusting the micrometer. To eject the extra sample liquid, spacer, and adjacent PTFE ring have holes. Inset: photograph of OPPC.

further correction. By using other connectors, further correction would be necessary to compensate for the electrical delay length from the reference plane, and deviation from ideal propagation in this calculation would cause some error. This direct connectivity of the APC7 improves the precision of the measurement especially in the $\mathrm{HF}$ region.

Details of the OPPC design are shown in Fig. 3 (a photograph of the OPPC is also shown in the inset of this figure). The spacer (viii) separates the two electrodes (ii, vi). Each electrode is held by two supporting quartz glass plates (i, v), which exhibit low electric conductivity and low thermal expansion. The quartz glass plates are essential for the measurement of temperature dependence. For the precise measurement at various temperatures, it is important to keep the separation of electrodes stable. By using quartz glass, the temperature dependence of the separation is suppressed at $3 \%$ for temperature change of $100 \mathrm{~K}$. If the measurement on temperature dependence is not of interest, the quartz glass can be replaced with a polymer isolator such as Teflon, and the cost of producing the cell can be decreased.

\section{EXPERIMENT}

Dielectric measurements were carried out from $40 \mathrm{~Hz}$ to $10 \mathrm{GHz}$. Three different measuring systems were used: an auto-balancing bridge (Agilent 4294 impedance analyzer) from $40 \mathrm{~Hz}$ to $110 \mathrm{MHz}$, the S-parameter method using two different network analyzers (Hewlett-Packard 4195A and Agilent 8510C) from $1 \mathrm{MHz}$ to $500 \mathrm{MHz}$ and from $100 \mathrm{MHz}$ to $10 \mathrm{GHz}$, respectively. The measured impedance and S-parameter (complex reflection coefficient) were transformed into complex permittivity by the lumped capacitance method, in which the DUT is treated as an infinitesimally small termination component. ${ }^{1}$ In the OPPC measurement, the DUT is connected to the end of a $0.3 \mathrm{~m}$-long coaxial cable. On the other hand, $1 \mathrm{~m}$-long four-terminal pair cables are used for the conventional parallel plate cell measurement. Most measurements were carried out at controlled room temperature $(293 \pm 1 \mathrm{~K})$. For the temperature dependence measurement, the temperature was controlled within $\pm 0.1 \mathrm{~K}$ by using a commercially available temperature chamber (Espec MC811T). 


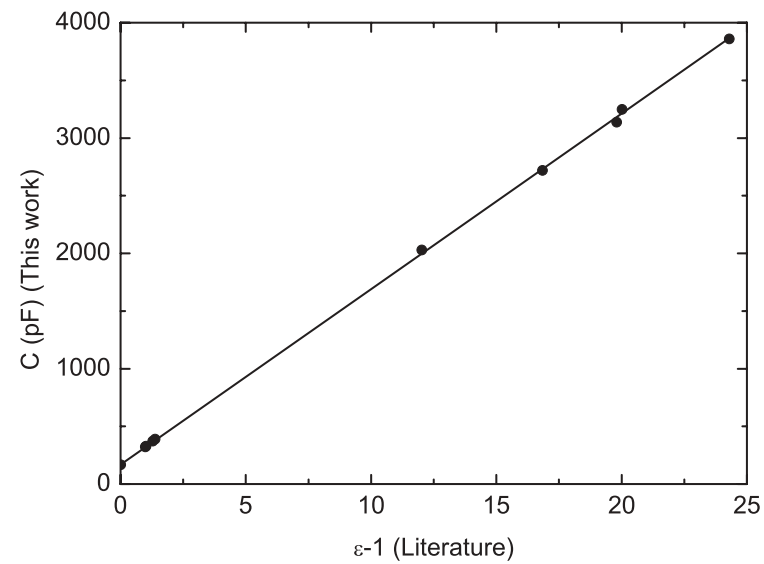

FIG. 4. Plot of capacitance measured at sufficiently low frequency against literature value of $\left(\varepsilon_{r}-1\right)$ for several liquid materials. Solid line represents result of least-squares fit with fixed interception value at the capacitance of air.

In order to demonstrate the comparison between the OPPC and conventional method, conventional parallel plate call was also used in the present paper. The gap separation of this cell was $520 \mu \mathrm{m}$ (when the spacer is used for liquid sample), and the diameter of the plates was $40 \mathrm{~mm}$. To remove the contribution from the stray capacitance, three-terminal method was employed. Theoretically calculated capacitance of this cell is $21.4 \mathrm{pF}$, whereas the measured value is $21.5 \mathrm{pF}$.

Liquid $n$-decane, toluene, 1-hexanol, and ethanol were purchased from Kishida Chemical Co., Ltd.; benzene, 1butanol, 1-propanol, and acetone were purchased from Kanto Chemical Co., Inc.; and $n$-dodecane was purchased from Merck-Schuchardt OHG. Polyethylene (PE) and polypropylene (PP) were provided by Kobe Steel Ltd., and polyethylene terephthalate (PET) was provided by Teijin Dupont. Commercially available polytetrafluoroethylene sheets produced by Nichias Corporation were used in this work. As measured with a micrometer, the thickness of the PE, PP, PET, PTFE, and paper films were found to be $90 \pm 1 \mu \mathrm{m}( \pm 1.1 \%)$, $90 \pm 1 \mu \mathrm{m}( \pm 1.1 \%), 84 \pm 1 \mu \mathrm{m}( \pm 1.2 \%), 72 \pm 1 \mu \mathrm{m}$ $( \pm 1.4 \%), 96 \pm 3 \mu \mathrm{m}( \pm 3.1 \%)$ and $92 \pm 1 \mu \mathrm{m}( \pm 1.1 \%)$, respectively. The water content of the paper was $5.60 \%$, which was measured by moisture meter (A\&D MX-50).

\section{RESULTS AND DISCUSSION}

Because the guard electrode that removes the effect of parasitic capacitor is not included in the OPPC, an estimation of parasitic capacitor is important for precise measurement. The capacitance of the sample cell and parasitic capacitor is given by the following equation:

$$
C=C_{0} \varepsilon_{r}+C_{f}=C_{0}\left(\varepsilon_{r}-1\right)+\left(C_{0}+C_{f}\right),
$$

where $C, C_{0}, C_{f}$, and $\varepsilon_{r}$ are the total capacitance, the capacitance of the sample, that of the fringing field and the dielectric permittivity of the sample. By plotting the measured $C$ against the literature values $\left(\varepsilon_{r}-1\right)$ for several materials, $C_{0}$ and $C_{f}$ can be estimated from the slope and intercept of the regression line. Figure 4 shows the relationship between the measured $C$ and the literature values for air, $n$-decane, $n$-dodecane,

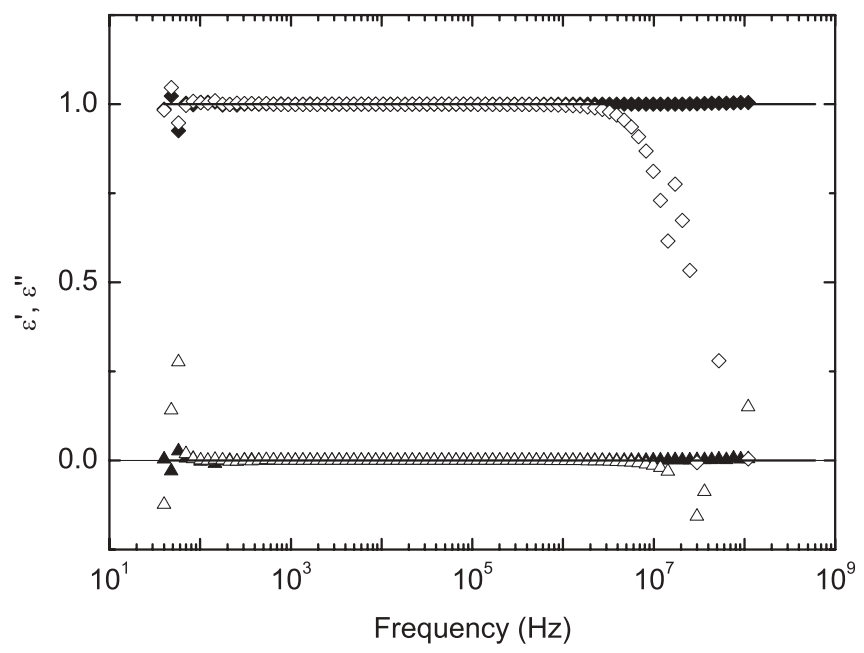

FIG. 5. Complex permittivity of air measured with OPPC (solid symbols) and conventional parallel plate cell (open symbols). Diamonds and triangles stand for permittivity and dielectric loss, respectively. Solid curve shows ideal value.

benzene, toluene, 1-hexanol, 1-butanol, 1-propanol, acetone, and ethanol. ${ }^{15}$ The solid line shows the result of the leastsquares fit where the intercept is fixed at $167.9 \mathrm{pF}$, which is the $C$ value of air. The data of the examined materials are well described by a linear function. The evaluated $C_{0}$ and $C_{f}$ values are $152.2 \mathrm{pF}$ and $15.7 \mathrm{pF}$, respectively. Complex permittivity presented below is calculated using these values.

To check the validity of the OPPC measurement and to conduct a comparison with conventional measurements, several well-known materials are examined. Figures 5-8 show the complex permittivity of air, benzene, hexanol, and ethanol measured by using the OPPC and conventional parallel plate capacitor. The former and the latter are represented by solid and open symbols, respectively. Solid curves in these figures represent the reported complex permittivity at $20^{\circ} \mathrm{C}$ (Ref. 15 for benzene, Ref. 16 for hexanol, and ethanol). Dielectric permittivity of these materials covers a wide range from 1 to 30 .

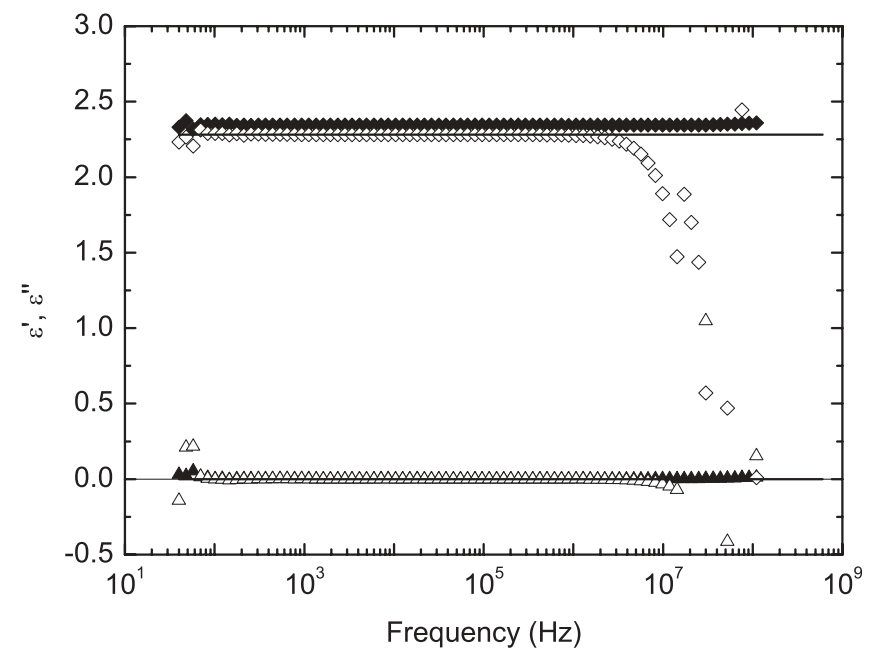

FIG. 6. Complex permittivity of benzene measured with OPPC (solid symbols) and conventional parallel plate cell (open symbols). Diamonds and triangles stand for permittivity and dielectric loss, respectively. Solid curve shows literature value at $20^{\circ} \mathrm{C} .{ }^{15}$ 


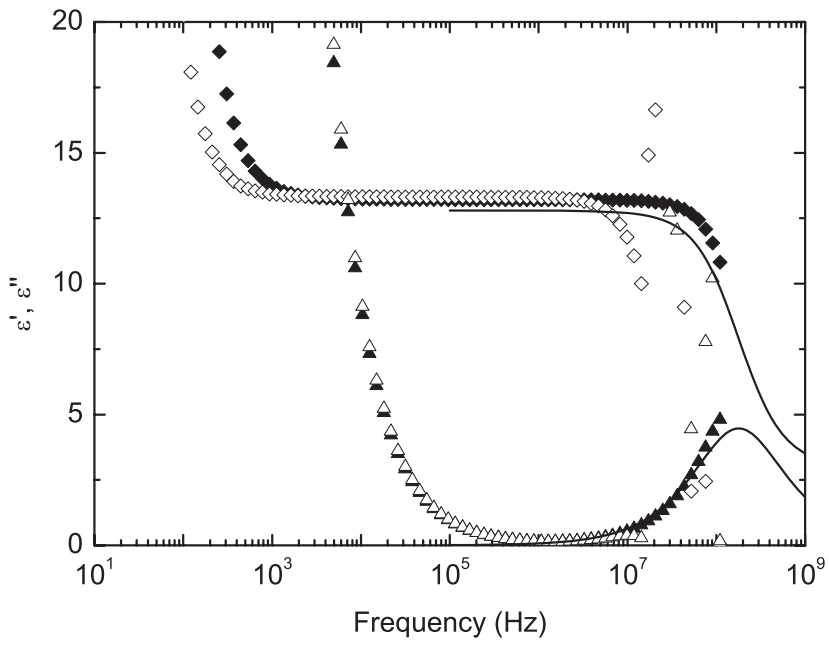

FIG. 7. Complex permittivity of 1-hexanol measured with OPPC (solid symbols) and conventional parallel plate cell (open symbols). Diamonds and triangles stand for permittivity and dielectric loss, respectively. Solid curve shows literature value at $20^{\circ} \mathrm{C} .16$

For all materials, the loci of OPPC are in close agreement with the known literature values over the observed frequency range. On the other hand, the values observed using the conventional capacitor deviate from the literature values above $1 \mathrm{MHz}$ due to effect from the low frequency side of resonance. The data for hexanol and ethanol show large contributions from electrode polarization in the LF region. The electric polarization obtained from using the OPPC and the conventional capacitor are not consistent because the electrode polarization generally depends on the gap separating the electrodes and on the electrode material. ${ }^{17-19}$ To evaluate the difference in the accuracy between OPPC and the conventional capacitor, the absolute value of the loss tangent, $\tan \delta$, of air is also presented in Fig. 9. Because ideally the $\tan \delta$ of air is zero, the observed $\tan \delta$ represents the inaccuracy of the measurement. Above $1 \mathrm{MHz}$, the magnitude of $\tan \delta$ in the conventional capacitor increases drastically with increasing frequency, whereas that



FIG. 8. Complex permittivity of ethanol measured with OPPC (solid symbols) and conventional parallel plate cell (open symbols). Diamonds and triangles stand for permittivity and dielectric loss, respectively. Solid curve shows literature value at $20^{\circ} \mathrm{C} .{ }^{16}$

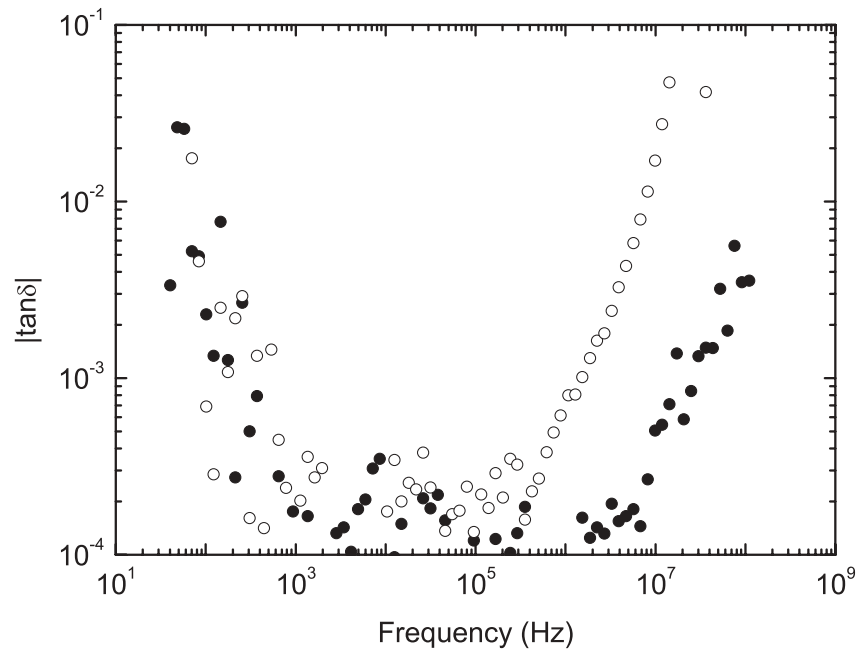

FIG. 9. Absolute value of $\tan \delta$ for air versus frequency plotted on doublelogarithmic scales.

of the OPPC remains below 0.01 over the full frequency range observed. The data are also scattered in the frequency region below $1 \mathrm{kHz}$. This is due to the lack of capacitance of the DUT. This would be reduced by increasing the capacitance.

The parallel plate configuration of the OPPC is especially suitable for film samples. Recently, the frequency range used in electronic devices has been increasing. The OPPC measurement in the HF region should also provide a good solution in this regard. The obtained complex permittivity for several polymer films is shown in Fig. 10-14. The complex permittivity measured with the OPPC is shown by solid symbols, and the complex permittivity measured with a conventional parallel plate cell is shown by open symbols. In the LF region (below $1 \mathrm{MHz}$ ), the complex permittivity values measured by using both methods are in agreement. The magnitudes of the deviation from conventional values are lower by $1.5 \%$ for PE, $1 \%$ for PP, $2 \%$ for PET, $2 \%$ for PTFE, and 3\% for paper samples, respectively. This may be attributed by roughness in the film thickness (less than $4 \%$ ) and precision of the cell

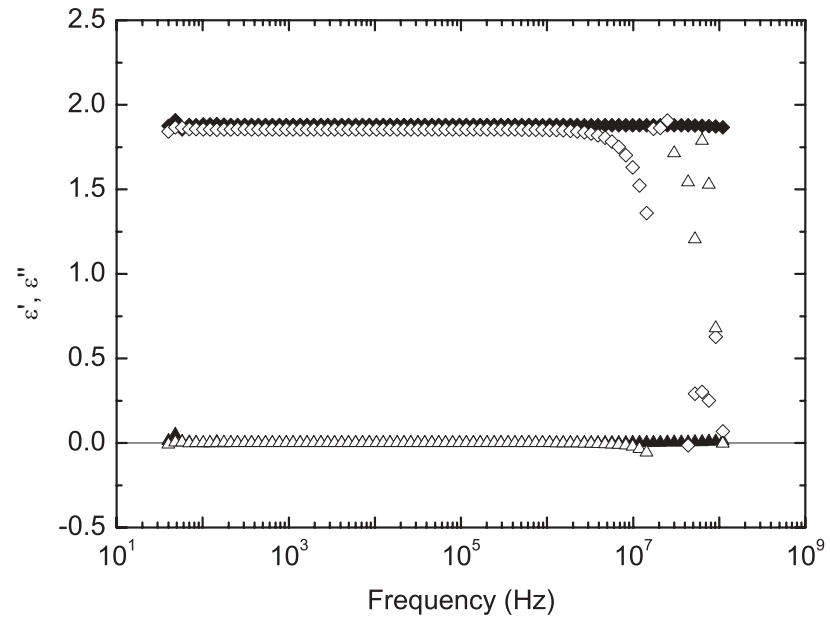

FIG. 10. Complex permittivity of PE measured with OPPC (solid symbols) and conventional parallel plate cell (open symbols). Diamonds and triangles stand for permittivity and dielectric loss, respectively. 


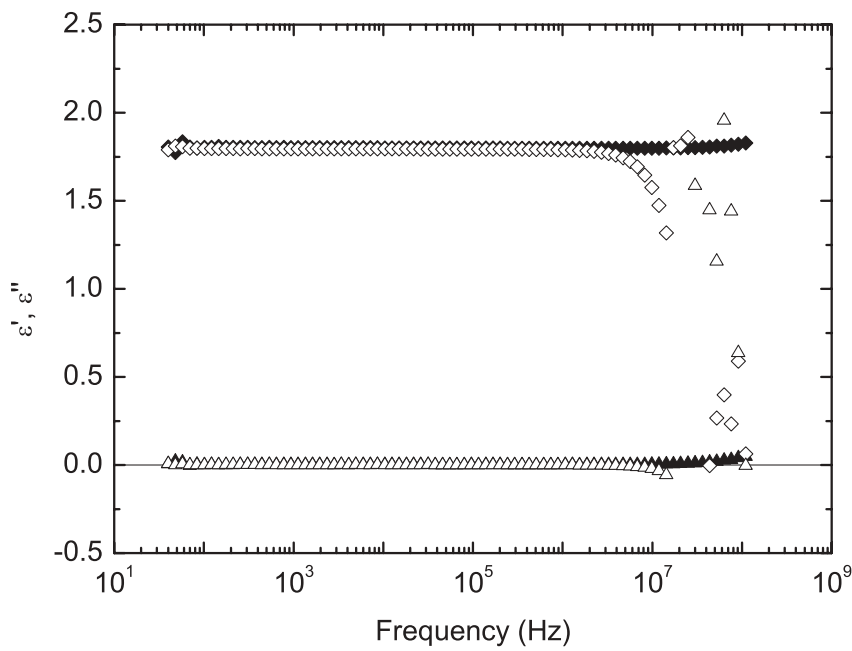

FIG. 11. Complex permittivity of PP measured with OPPC (solid symbols) and conventional parallel plate cell (open symbols). Diamonds and triangles stand for permittivity and dielectric loss, respectively.

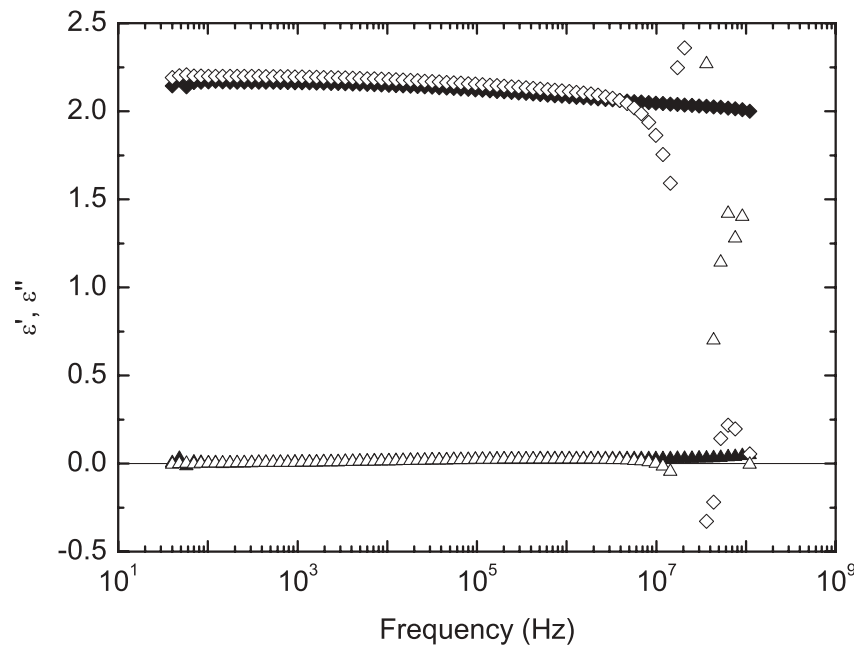

FIG. 12. Complex permittivity of PET measured with OPPC (solid symbols) and conventional parallel plate cell (open symbols). Diamonds and triangles stand for permittivity and dielectric loss, respectively.

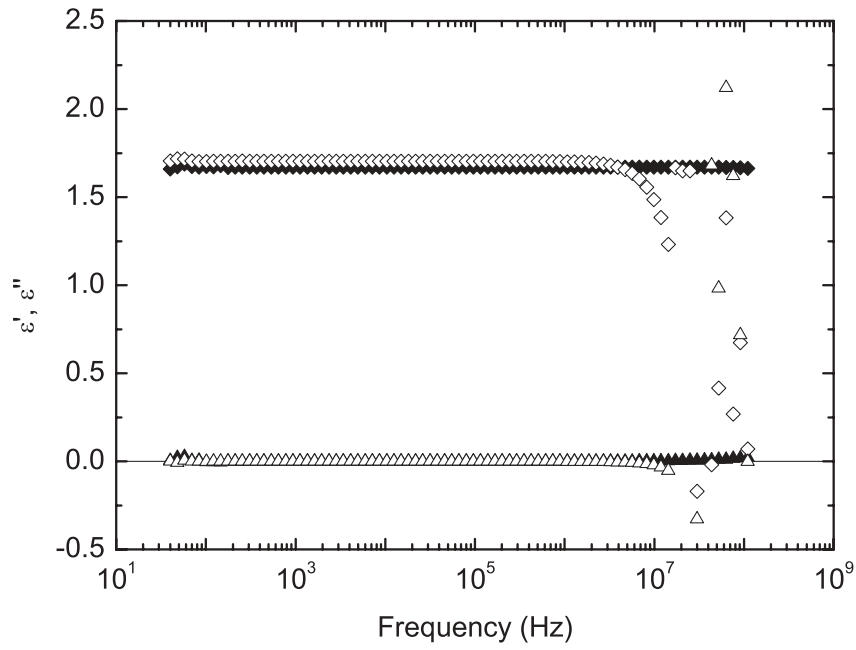

FIG. 13. Complex permittivity of PTFE measured with OPPC (solid symbols) and conventional parallel plate cell (open symbols). Diamonds and triangles stand for permittivity and dielectric loss, respectively.

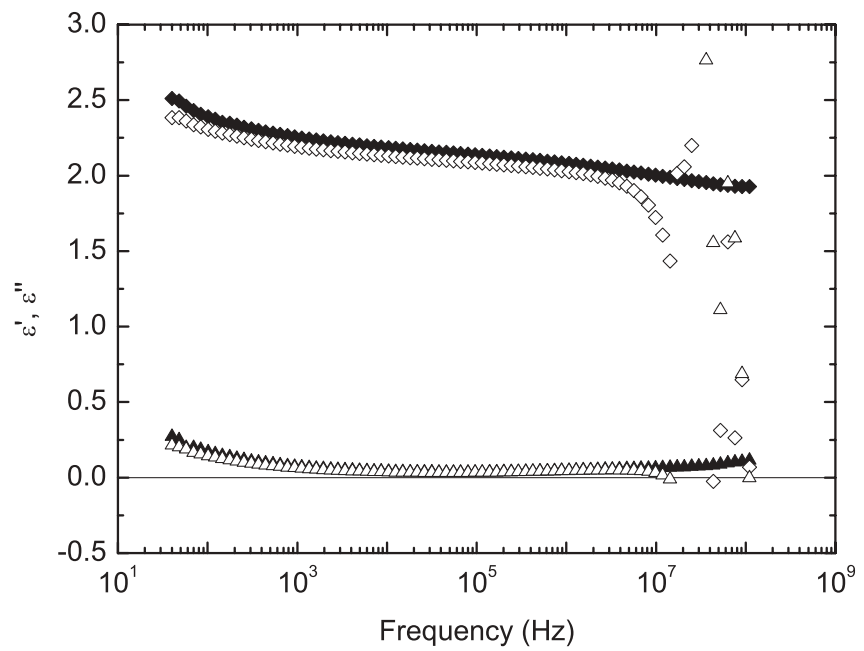

FIG. 14. Complex permittivity of paper measured with OPPC (solid symbols) and conventional parallel plate cell (open symbols). Diamonds and triangles stand for permittivity and dielectric loss, respectively.

assembly (less than $2 \%$ in the value of capacitance). Similar to the result for the liquid samples, the complex permittivity in the HF region obtained by the conventional method exhibited crucial deviation from that in the LF region. On the other hand, the complex permittivity from OPPC stays at a reasonable value even in the HF region. It should be noted that in the present experiment, the film samples were examined without evaporation of the electrode onto the sample, and the sample was simply sandwiched between the two electrodes of the OPPC. Therefore, in addition to the advantage of wide frequency range, the complex permittivity of polymer films can be evaluated in a straightforward manner.

Because the OPPC has a coaxial connection to the instrument, it can also be used for the reflectometry method using a network analyzer. Figure 15 shows the complex permittivity of air obtained by two network analyzers with different frequency ranges, HP 4195 for the lower frequency region (solid

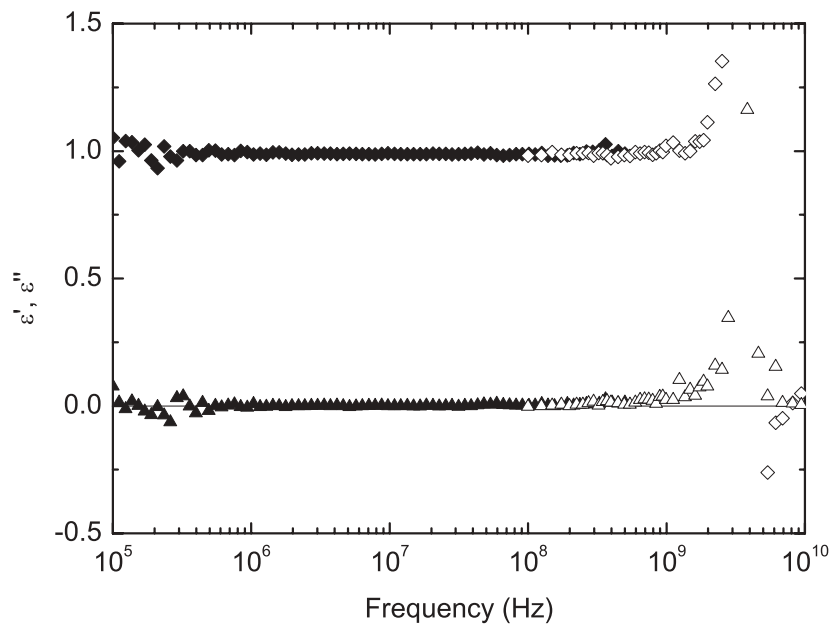

FIG. 15. Complex permittivity of air measured using OPPC with two different network analyzers. Solid diamonds, solid triangles, open diamonds, and open triangles represent the permittivity measured with HP4195, dielectric loss measured with HP4195, permittivity measured with Agilent 8510, and dielectric loss measured with Agilent 8510, respectively. 
symbol) and Agilent 8510 for the higher frequency region (open symbol), respectively. The OPPC exhibits good performance over the entire megahertz region. At about $4 \mathrm{GHz}$, both permittivity and dielectric loss sharply increase, owing to resonance. The frequency dependence of the magnitude of complex reflection coefficient (not shown in this paper) shows a sharp absorption peak at $7.5 \mathrm{GHz}$. Within the framework of LC (or LCR) resonance, such a sharp absorption peak should not be present in the complex reflection coefficient. Nozaki and coworkers have pointed out that it is important to take into account multiple reflections within the radial direction of the parallel plate capacitor above $1 \mathrm{GHz} .{ }^{14}$ Within this framework, the complex reflection coefficient can be derived from a plane wave approximation. According to this calculation method, the resonance frequency can be estimated at 7.0 GHz. A more precise estimation can be obtained by considering the circular shape of the OPPC using a Bessel function. This yields a resonance frequency of $7.5 \mathrm{GHz}$, which perfectly coincides with the experimental value within the experimental resolution. It should be noted that the resonance frequency depends on the diameters $a$ and $b$, and on the complex permittivity $\varepsilon_{r}^{*}$, and is independent of the gap separating the plates, $d$, on the basis of these calculation.

In all method described here, the resonance frequency increases with increasing diameter. For HF measurements, therefore, the diameter should be smaller whereas for LF measurements, the diameter should be large. This causes a tradeoff between the quality of HF and LF measurements. The value of the outer diameter should be chosen considering the frequency range where the OPPC is used.

\section{CONCLUSIONS}

A new configurational, the OPPC, has been proposed. Considering propagation properties, its structure is topologically equivalent to that of a coaxial cable. This improves the performance during HF measurements while maintaining high accuracy in the LF region; therefore, the applicable frequency region of the OPPC is enlarged. The OPPC enables a new method for wide frequency measurements of liquid and film samples. Some examples for these measurements are presented for several materials, and all the measurement results are in good agreement with the literature values. The OPPC is also applicable to reflectometry methods, such as the Sparameter method using a network analyzer. The OPPC can provide complex permittivity over a wide frequency region in a single sweep. This advantage will possibly be useful for investigating on time-dependent processes such as crystallization and aging.

\section{ACKNOWLEDGMENTS}

We wish to thank Dr. Z. Chen for many valuable discussions. This work was partly supported by KAKENHI Grant No. 19340116, Grant-in-Aid for Scientific Research (B), from the Ministry of Education, Culture, Sports, Science and Technology (MEXT) of Japan.

${ }^{1}$ F. Kremer, A. Schönhals, Broadband Dielectric Spectroscopy (Springer, Berlink, 2002)

${ }^{2}$ Wayne Kerr Electronics, 6500B Series Technical data sheet.

${ }^{3}$ Agilent Technologies, 4294A Data Sheet.

${ }^{4}$ Solartron, 1260 Data Sheet.

${ }^{5}$ N. G. McCrum, B. E. Read, and G. Williams, Anelastic and Dielectric Effects in Polymeric Solids (Wiley, New York, 1967).

${ }^{6}$ F. Kremer, J. Non-Cryst. Solids 305, 1 (2002).

${ }^{7}$ C. M. Roland, M. J. Schroeder, J. J. Fontanella, and K. L. Ngai, Macromolecules 37, 2630 (2004).

${ }^{8}$ K. Grzybowska, A. Grzybowski, J. Zioło, and M. Paluch, J. Chem. Phys. 125, 044904 (2006).

${ }^{9}$ H. Wagner, and R. Richert, J. Chem. Phys. 110, 11660 (1999).

${ }^{10}$ P. Lunkenheimer, and A. Loidl, Chem. Phys. 284, 205 (2002).

${ }^{11}$ A. Puzenko, Y. Hayashi, Y. E. Ryabov, I. Balin, Y. Feldman, U. Kaatze, and R. Behrends, J. Phys. Chem. B, 109, 6031 (2005).

${ }^{12}$ N. Shinyashiki, D. Imoto, and S. Yagihara, J. Phys. Chem. B 111, 2181 (2007).

${ }^{13}$ G. Schaumburg, Dielectric Newsletter 1, 4 (1994).

${ }^{14}$ K. Murata, A. Hanawa, and R. Nozaki, J. Appl. Phys. 98, 084107 (2005).

${ }^{15}$ Handbook of Chemistry: Pure Chemistry, 5th ed., edited by Y. Iwasawa et al. (Maruzen Co., Ltd., Tokyo, 2004).

${ }^{16}$ P. Petong, R. Pottel, and U. Kaatze, J. Phys. Chem. A 103, 6114 (1999).

${ }^{17}$ R. J. Klein, S. Zhang, S. Dou, B. H. Jones, R. H. Colby, and J. Runt, J. Chem. Phys. 124, 144903 (2006).

${ }^{18}$ J. R. Macdonald, Phys. Rev. 92, 4 (1953).

${ }^{19}$ A. D. Hollingsworth, and D. A. Saville, J. Colloid Interface Sci. 257, 65 (2003) 